\title{
QoS-Aware Reference Station Placement for Regional Network RTK
}

\author{
Maolin Tang ${ }^{1}$ \\ ${ }^{1}$ Cooperative Research Centre for Spatial Information, Faculty of Science and Technology, Queensland University of Technology \\ 2 George Street, Brisbane, Australia \\ Email: m.tang@qut.edu.au
}

Received December $24^{\text {th }}, 2008$; revised January $30^{\text {th }}, 2009$; accepted February $26^{\text {th }}, 2009$.

\begin{abstract}
Network RTK (Real-Time Kinematic) is a technology that is based on GPS (Global Positioning System) or more generally on GNSS (Global Navigation Satellite System) measurements to achieve centimeter-level accuracy positioning in real-time. Reference station placement is an important problem in the design and deployment of network RTK systems as it directly affects the quality of the positioning service and the cost of the network RTK systems. This paper identifies a new reference station placement for network RTK, namely QoS-aware regional network RTK reference station placement problem, and proposes an algorithm for the new reference station placement problem. The algorithm can always produce a reference station placement solution that completely covers the region of network RTK.
\end{abstract}

Keywords: Reference Station, Placement, Regional Network RTK, QoS

\section{Introduction}

Network RTK (Real-Time Kinematic) is a technology that is based on GPS (Global Positioning System) or more generally on GNSS (Global Navigation Satellite System) measurements to achieve centimeter-level accuracy positioning in real-time. It involves a network of reference stations that are used to take and transmit their raw GPS or GNSS measurements [1,2,3,4].

The success of network RTK in recent years has resulted in the establishment of network RTK positioning services to anyone who is willing to pay for them. In order to provide network RTK services to an area, such as a state or a country, many reference stations must be set up and maintained. However, setting-up and maintaining reference stations is costly, typically tens of thousands of Australian dollars each with annual operational cost of approximately $10 \%$ of the reference station cost. Therefore, it is desirable to minimize the total number of reference stations without compromising the QoS of network RTK. However, the reference station placement problem has not drawn enough attention from the community, and little research has been done.

In our preliminary research, we have identified a significant reference station placement problem, namely location-oriented reference station placement problem, and proposed an effective and efficient algorithm for the reference station placement problem [5]. That reference station placement problem is described as: Given a set of potential locations where reference stations can be set up and the locations of the users, select reference station locations among the potential reference station locations such that the total number of reference stations is minimum subject to a constraint, that is, the maximal distance between any user to the closest reference station does not exceed a parameter $D_{\max }$. This maximum distance constraint must be satisfied in order to guarantee the accuracy of the positioning services.

Recently, we identified and proposed an algorithm for an area-oriented reference station placement problem for network RTK [6]. Given a service area that a network RTK needs to cover, the area-oriented reference station placement problem strives to find a reference placement such that the service area is completely covered by the reference stations. The major deficiency of the algorithm is that it cannot provide a reference placement solution that can guarantee to provide QoS because the algorithm cannot guarantee to generate a reference station placement solution that completely covers the service area although in most cases it does. As a result, those users who are located in an area where is not covered by the reference stations may not receive quality positioning service.

In this paper we propose a new reference station placement problem, namely, QoS-aware regional network RTK reference station problem, and extend the area-oriented reference station placement problem to guarantee to generate a reference station placement solution that covers the whole service area. The proposed algorithm has been implemented and tested by simulation. Experimental results show that the algorithm is efficient and effective. 
The remaining paper is organized as follows. Section 2 formulates the QoS-aware regional network RTK reference station placement problem. Section 3 discusses related work. Section 4 presents our approach to the new reference station placement problem in detail. The simulation results are presented in Section 5. Finally we conclude the proposed approach in Section 6.

\section{Problem Formulation}

Given an area and the maximal distance constraint from any user to its closest reference station, the QoS-aware regional network RTK reference station placement problem is to find locations on the area such that all the users at any location within the area can receive real-time centimeter accuracy positioning services.

In order to guarantee a user can get the real-time centimeter accuracy positioning services, it must be guaranteed that the maximum distance between the user to its closest reference station is less than or equals to the maximum distance constraint. Because the user can be distributed at any position on the area, the maximum distance constraint implies that the maximum distance between any position in the area and its closest reference station must not exceed the maximum distance constraint. Thus, the QoS-aware network RTK reference station placement is formulated as:

Given an area $A$ and the maximal distance constraint $D_{\max }$, find a set of reference stations $R=\left\{r_{1}, r_{2}, \ldots, r_{n}\right\}$ such that $|R|$ is minimum, subject to $\forall p=\left(x_{p}, y_{p}\right) \in A, \quad \exists r_{i}=\left(x_{i}, y_{i}\right) \in R$, where, and $\sqrt{\left(x_{p}-x_{r}\right)^{2}+\left(y_{p}-y_{r}\right)^{2}} \leq D_{\max }$. The constraint guarantees that the QoS, or the accuracy of positioning services, is satisfied.

\section{Related Work}

In our preliminary research on network RTK, we have identified a so-called location-oriented reference station placement problem. The location-oriented reference station placement problem is stated as below:

Given a set of reference station candidates $R=\left\{r_{1}, r_{2}, \ldots, r_{n}\right\}$, a set of users $U=\left\{u_{1}, u_{2}, \ldots, u_{\mathrm{m}}\right\}$, and the maximal distance between any user and its closest reference station $D_{\max }$, the location-oriented reference stations placement problem is to find $R_{0} \subseteq R$, such that $\left|R_{0}\right|$ is minimal, subject to $\forall u_{i}=\left(x_{i}, y_{i}\right) \in U, \exists r_{j}=\left(x_{j}, y_{j}\right) \in R$ and $\sqrt{\left(x_{i}-x_{j}\right)^{2}+\left(y_{i}-y_{j}\right)^{2}} \leq D_{\max }$, where $1 \leq i \leq m$ and $1 \leq j \leq n$.

The location-oriented reference stations placement problem can be represented in a so-called graph $G=(V, E)$, where $V=U \cup R$. An edge $\left(v_{1}, v_{2}\right) \in E$, if and only if $v_{1}=\left(x_{1}, y_{1}\right) \in U, \quad v_{2}=\left(x_{2}, y_{2}\right) \in R$, and $\sqrt{\left(x_{1}-x_{2}\right)^{2}+\left(y_{1}-y_{2}\right)^{2}} \leq D_{\max }$, where $\left(x_{1}, y_{1}\right)$ and $\left(x_{2}, y_{2}\right)$ represent the location of $v_{1}$ and the location of $v_{2}$, respectively. Therefore, the location-oriented reference station placement problem can be transformed into the following graph-theoretic problem:

Given a RTK graph $G=(V, E)$, where $V=U \cup R$, find $R_{0} \subseteq R$, such as that $V=V_{0}$, where $V_{0}=\left\{v \mid(r, v) \in E \& r \in R_{0}\right\}$.

A RTK graph can be represented by an $m \times n$ adjacency matrix $A=\left[a_{i j}\right]_{m \times n}$, where

$$
a_{i j}= \begin{cases}1, & \text { if }\left(u_{i} \cdot r_{j}\right) \in E \\ 0, & \text { otherwise }\end{cases}
$$

For example, for the RTK graph shown in Figure 1. The adjacent matrix

$$
A=\left(\begin{array}{llll}
1 & 1 & 0 & 0 \\
0 & 0 & 1 & 1 \\
1 & 1 & 0 & 0 \\
0 & 1 & 1 & 1 \\
0 & 0 & 0 & 1
\end{array}\right)
$$

The location-oriented reference stations placement problem is a constrained version of the problem of finding a minimum dominating set of an arbitrary graph, which is an NP-hard [7].

It is conjectured that this reference stations placement problem is also NP-hard. Thus, we proposed a heuristic algorithm in [5].

The heuristic algorithm starts with an initial reference station placement that uses all the reference station candidates. Then, the number of reference station candidates is gradually reduced by iteratively removing redundant reference stations. A reference station is redundant if all the users that can be covered by the reference station can also be covered by other reference stations. A user is said to be covered by a reference station if the distance between the user and the reference station does not exceed $D_{\max }$. A redundant reference station $r_{i}$ can be identified in the matrix representation by checking if there is a second non-zero entry in the $j^{\text {th }}$ column of the matrix. Algorithm III is the high-level description of the location-oriented reference station placement algorithm.

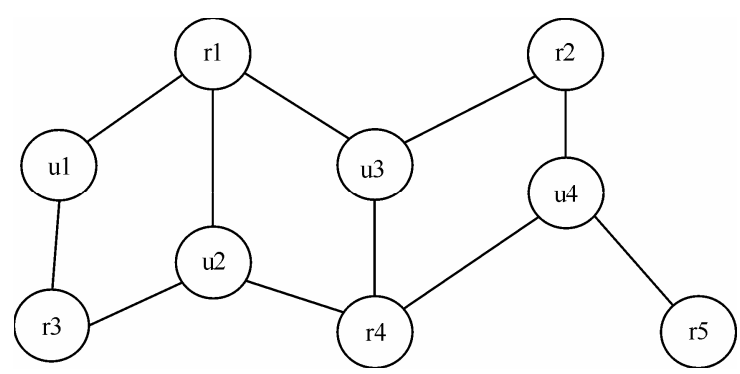

Figure 1. A RTK graph 
Algorithm 1: Location-oriented placement algorithm Require: Locations of reference station candidates, locations of users and the maximal distance allowed between a user and its reference station $D_{\max }$

Ensure: Return the location of reference stations generate its RTK graph $G=(V, E)$;

for each reference station candidate $r \in R$ do if $r$ is redundant then remove it from $R$; remove its associated edges from $E$; end if

end for

Output the location of the reference station candidates left in $R$.

It has been proved in [5] that the computational complexity of the algorithm is $O\left(n^{3} * m\right)$, where $m$ is the number of reference station candidates and $n$ represents the number of users. Details about the algorithm analysis can be found in [5].

\section{Approach}

The QoS-aware network RTK reference station placement problem is a continuous optimization problem and it is difficult to be tackled directly. Thus, we transform the QoS-aware network RTK reference station placement problem into the location-oriented reference station placement problem discussed in the Related Work, and use the location-oriented reference station placement algorithm to tackle the QoS-aware network RTK reference station placement problem.

In the following, we will discuss how to transform the QoS-aware network RTK reference station placement problem into the location-oriented reference station placement problem. Then, we will present an algorithm for the QoS-aware network RTK reference station placement problem, and then we analyses the optimality and efficiency of the new algorithm.

\subsection{Transformation}

The QoS-aware network RTK reference station placement problem is a continuous optimization problem, where the locations of reference stations are on a continuous two-dimensional region. Thus, it is difficult to be handled directly as its search space is infinite. However, the continuous optimization problem can be transformed into the location-oriented reference station placement problem using the following procedure.

First of all, a two-dimensional grid graph is generated to completely cover the whole region of the network RTK $A$. This idea is illustrated in Figure 2. In the figure, the filled enclosed area represents the network RTK service area A.

Then, we select those grid nodes on or outside the boundary such that the area of the polygon formed by connecting those grid nodes is minimal, but can completely cover the network RTK service area $A$. Figure 3 shows such a polygon based on the grid graph shown in Figure 2.

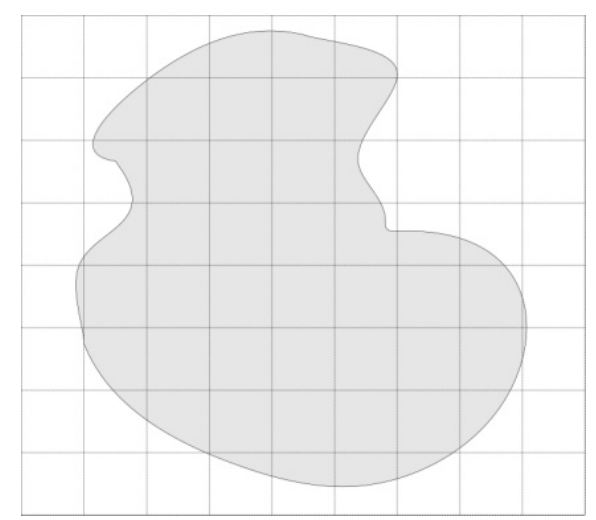

Figure 2. A grid graph that completelly covers the network RTK service area $A$

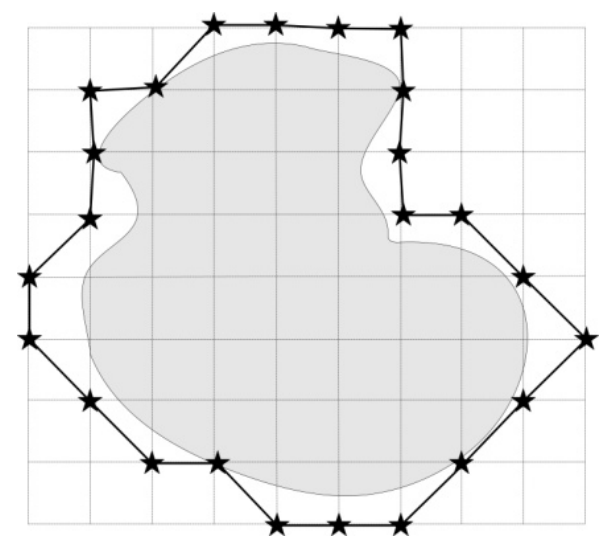

Figure 3. A minimal polygon that completelly covers the network RTK service area $\boldsymbol{A}$

And then, we create a sub-graph of the grid graph by selecting all the grid nodes and arcs enclosed by the polygon. Figure 4 shows the sub-graph generated from the grid graph and the polygon shown in Figure 3.

In order to make use of the location-oriented reference station placement algorithm discussed in the Related Work section, we need to create dummy users and initial reference stations (reference station candidates). The selection of users and initial reference stations is very important because if they are not well chosen, then the location-oriented reference station placement algorithm may not produce a solution that can completely cover the whole service area of the regional network RTK. Thus, in the following, we will introduce rules for selecting dummy users and initial reference stations. We will prove that by selecting dummy users and initial reference stations it is guaranteed that the algorithm can always produce a solution that can completely cover the given service area of the regional network RTK.

Given a grid graph $G=(V, E)$, all the nodes whose degree is greater than one are selected as reference stations and on each edge we create three dummy users: one at each end of the edge and one at the middle of the edge. The rules for selecting initial reference stations and the rules for selecting dummy users are illustrated in Figure 5 
and Figure 6, respectively. It will be proven in the paper that by selecting reference station candidates and user positions using the above rules, our algorithm can always produce a reference placement solution that can $100 \%$ covers the given service area $A$.

Using the rules, we can create a set of initial reference stations $R=\left\{r_{1}, r_{2}, \ldots, r_{n}\right\}$ and a set of users $U=\left\{u_{1}, u_{2}, \ldots, u_{\mathrm{m}}\right\}$, and have transformed the QoS-aware regional network RTK reference station problem into the location-oriented reference station placement problem.

\subsection{Algorithm Description}

Once we have transformed the QoS-aware regional network RTK reference station placement problem into the location-oriented reference station placement problem, we can make use of the ideas of the location-oriented reference station placement algorithm presented in the Related Work section to tackle the QoS-aware regional network RTK reference station placement problem. Algorithm 2 gives a high-level description of the algorithm for the QoS-aware regional network RTK reference station placement problem.

Algorithm 2: QoS-aware regional network RTK placement station placement algorithm

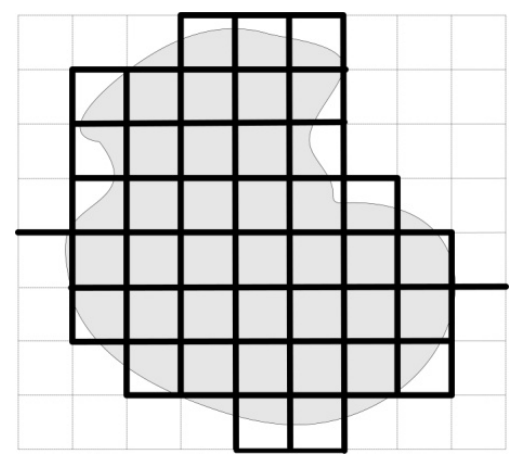

Figure 4. The subgraph enclosed by the minimal polygon

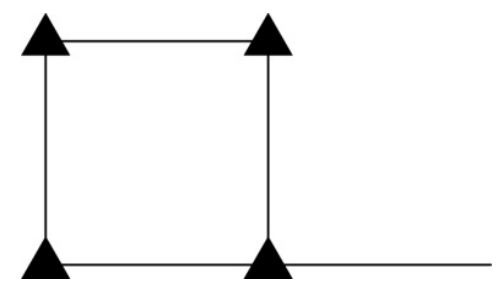

Figure 5. Initial reference stations selection rule

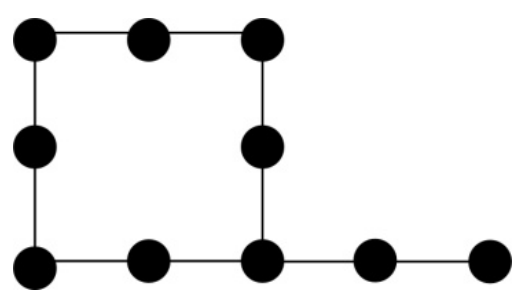

Figure 6. Dummy users selection rule
Require: Network RTK service area A and the maximal distance allowed between a user and its reference station $D_{\max }$.

Ensure: Return the location of reference stations generate a grid graph of grid size $\mathrm{g}$; generate the minimal polygon covering the network RTK service area $A$; generate the sub-graph enclosed by the minimal polygon;

use the rules to create reference station candidate set $R$ and user set $U$; generate its RTK graph $G=(U \cup R, E)$;

for each reference station candidate $r \in R$ do

if $r$ is redundant then remove it from $R$; remove its associated edges from $E$; end if

end for

Output the location of the reference station candidates left in $R$.

\subsection{Algorithm Analysis}

This subsection provides theoretic analysis of the algorithm for the QoS-aware regional network RTK reference station placement problem presented in the previous subsection.

Lemma 1: Algorithm 2 always produces a reference placement station placement solution in which every generated user is covered by at least one of the reference stations.

Proof: Algorithm 2 starts with generating a set of users and a set of reference stations, and then iteratively reduces redundant reference stations. It can be guaranteed by the rules for identifying users and initial reference stations that every generated user can be covered by at least reference station initially, and that during the iterative process of reducing redundant reference stations only redundant reference stations are removed, which means all the users that is covered by a removed redundant reference station can also be covered by another reference station. Thus, after removing all the redundant reference stations, every generated user is covered by at least one of the reference stations.

Theorem 1: Given an area $A$ and the maximum distance constraint $D_{\max }$. algorithm 2 always produces a reference station placement solution that completely covers $A$.

Proof: The given area A is enclosed by a polygon. If we can prove that the polygon is completely covered by the reference station placement solution produced by Algorithm 2, then we have proved that the given area $A$ is completely covered by the solution. In addition, it has been proved in Lemma 1 that Algorithm 2 can always produce a solution that covers all the dummy users. Thus, all we need to prove in the following is that when all the dummy users are covered, the polygon is $100 \%$ covered. 
The area of the enclosing polygon is composed of two basic types of two-dimensional areas, squares and isosceles right triangles. For each of the squares as shown in Figure 7 , it will be completely covered by the solution because in order to cover all the eight user position on the square as shown in Figure 6 at least two diagonal reference station candidates must be selected. When any two of the diagonal candidates on a square are selected, the whole area of the square will be covered. Note that the coverage of the reference station is always the length of the square. Thus, it can be concluded that all the square areas will be covered by the solution. Figure 7 illustrates the idea. In the figure, two diagonal candidates $\mathrm{A}$ and $\mathrm{C}$ are selected. The shadow circles display the coverage of the reference stations at candidate $\mathrm{A}$ and candidate $\mathrm{C}$.

For each of the isosceles right triangles as shown in Figure 8 , it will also be completely covered by the solution because according to the reference station candidate and user selection rules, the only possibility to cover all the three users on the line from $\mathrm{A}$ to $\mathrm{B}$ is to select the reference station candidate at position $\mathrm{A}$, and when that reference candidate is selected, the isosceles right triangle $\mathrm{ABC}$ will be completely covered by the reference station. This is illustrated in Figure 8.

The quality of solutions obtained by the algorithm and the computation time of the algorithm are both dependent

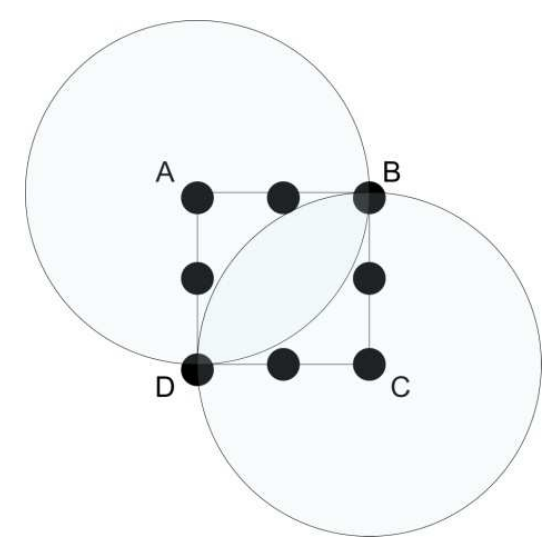

Figure 7. The square case

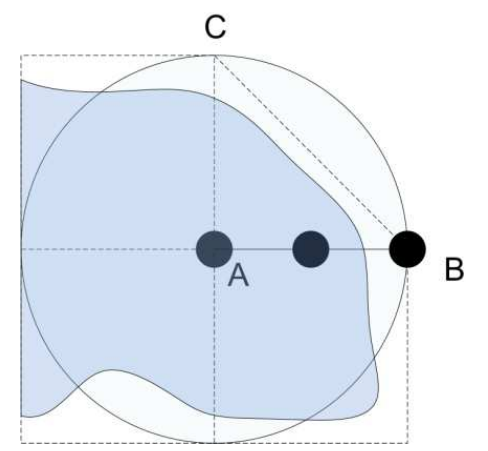

Figure 8. The isosceles right triangle case on the grid size $\mathrm{g}$, which is an important parameter used by the algorithm. In the following section, we present our empirical study results on the impact of $g$ on the optimality and performance of the algorithm.

\section{Experiment}

The proposed algorithm has been implemented in Microsoft Visual C\#. In order to test the optimality and performance of the algorithm, we used the implemented algorithm to find a reference station placement solution for the network RTK area shown in Figure 2. The value of $D_{\max }$. was fixed to $140 \mathrm{~km}$, but the values used for the gird size g varied from 10 to 140 with an increment of 10 . All experiments were conducted on desktop computers with an Intel Core 2 Duo $6300 \mathrm{CPU}(1.86 \mathrm{GHz})$ and $2 \mathrm{~GB}$ of RAM. Table I records the experimental results.

Figure 9 shows the trend of the quality of the solutions obtained by the algorithm when the grid size increases. It can be seen from the figure that the grid size used by the algorithm directly affects the quality of the solutions obtained by the algorithm, and that the smaller the grid size the better solution the algorithm produces. For example, when the grid size is 20 the solution produced by the algorithm needs only 23 reference stations. However, when the grid size increases to 140 the solution generated by the algorithm needs 34 reference stations.

Figure 10 shows the trend of the computation time of the algorithm when the grid size increases. It can be seen from the figure that the computation time of the algorithm decreases dramatically when the grid size increase. For example, when the grid size is 20 the computation time is 47.81 seconds. When the grid size increases to 140 the computation time is only 0.02 seconds.

The experimental results reveal that the smaller the grid size is, the better the quality of the solution is. However, the computation time increases very quickly when the grid size decreases. Figure 11 displays the result for the area-oriented station placement problem obtained by the implemented algorithm when the grid size $g=20$. In the figure, the small filled squares represent reference stations and the circles are the coverage of the corresponding reference stations.

Table 1. Experimental results on the impact of $g$ on the optimality and computation time of the algorithm

\begin{tabular}{ccc}
\hline $\begin{array}{c}\text { Grid Size } \\
(g)\end{array}$ & $\begin{array}{c}\text { Reference Station } \\
\text { Number }\end{array}$ & $\begin{array}{c}\text { Time } \\
\text { (second) }\end{array}$ \\
\hline 20 & 23 & 47.81 \\
40 & 26 & 2.32 \\
60 & 27 & 0.45 \\
80 & 28 & 0.15 \\
100 & 31 & 0.06 \\
120 & 33 & 0.03 \\
140 & 34 & 0.02 \\
\hline
\end{tabular}




\section{Conclusions}

This paper has identified a new network RTK reference station placement problem, namely QoS-aware regional network RTK reference station placement problem, and has proposed an effective algorithm for the problem.

The basic idea behind the algorithm is to transform the QoS-aware regional network RTK reference station placement problem, which is basically a continuous areaoriented reference station placement problem, into an existing discrete location-oriented reference station placement problem and make use of the existing algorithm for the location-oriented reference station placement problem to solve the challenging area-oriented reference station placement problem. The transformation process and the algorithm for the QoS-aware regional network RTK reference station placement problem have been discussed in detail in this paper.

The most challenging job in tackling the continuous area-oriented reference station placement problem is to guarantee the reference station placement solution can completely cover the region of the network RTK. It has been proved in this paper that our proposed algorithm can always produce a reference station placement solution that can $100 \%$ cover the service area of a regional network RTK.

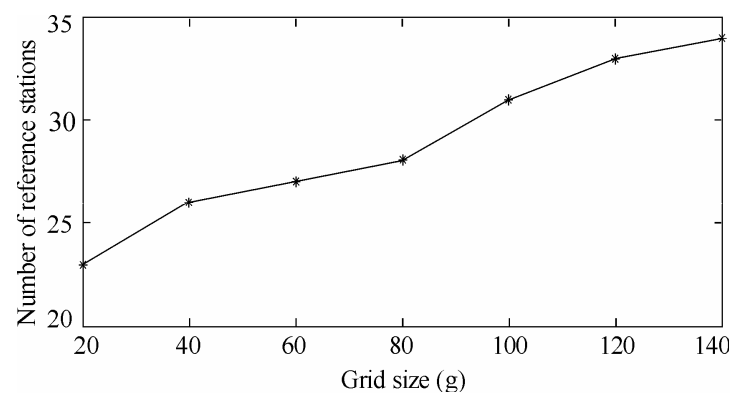

Figure 9. The impact of grid size on the optimality of the algorithm

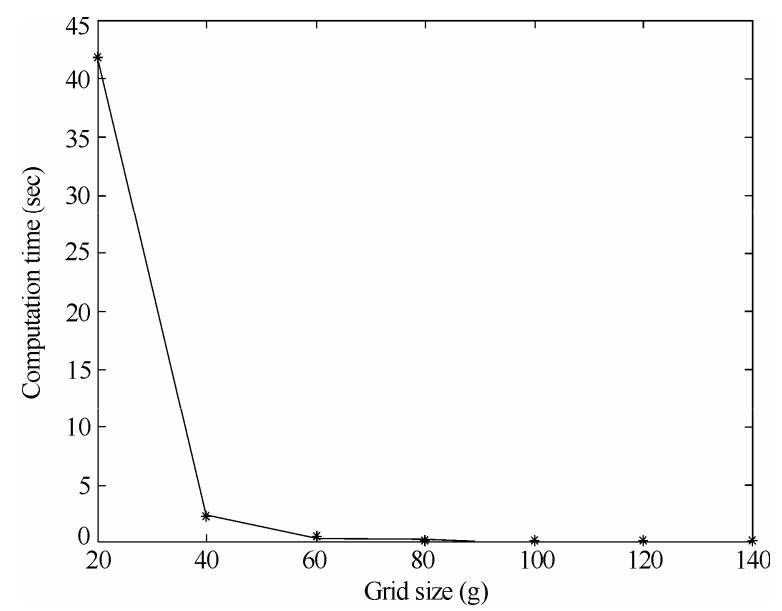

Figure 10. The impact of grid size on the computation time of the algorithm

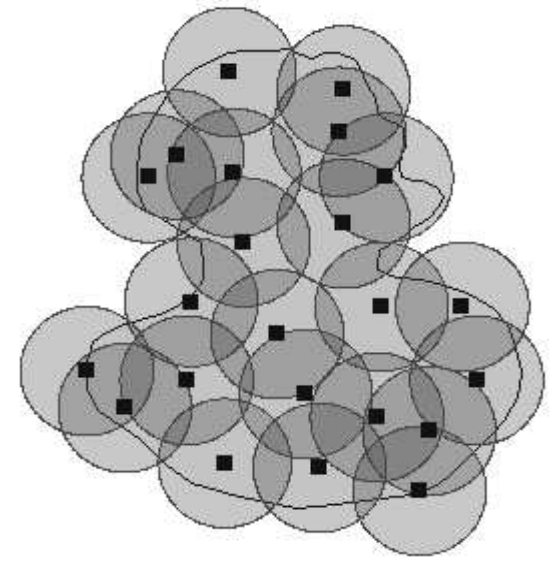

Figure 11. An area-oriented reference station placement solution

The grid size $g$ is an important control parameter in the transformation as it affects the optimality and performance of the algorithm. Generally, the smaller $g$ 's is, the better the solution is. However, when $g$ 's value becomes small, the computation time increases dramatically. A potential solution to this problem is parallel implementations of the algorithm.

\section{Acknowledgement}

The work was carried out with financial supports from Cooperative Research Centre for Spatial Information (CRCSI) project 1.4: "Integrating electricity, telecommunications and government infrastructure to deliver precise positioning services in regional areas", 2007-2010.

The proposed algorithm was implemented by Lifeng Ai of Queensland University of Technology.

\section{REFERENCES}

[1] L. Wanninger, "Introduction to Network RTK," http://www. networkrtk. info/intro/introduction.html.

[2] C. Rizos, "Network RTK research and implementation-A geodetic perspective," Journal of Global Positioning Systems, Vol. 1, No. 2, pp. 144-150, 2003.

[3] L. Wanninger, "GPS on the web: Virtual reference stations VRS," GPS Solutions, Vol. 7, pp. 143-144, 2003.

[4] G. Fotopoulos and M. E. Cannon, "An overview of multireference station methods for Cm-Level positioning," GPS Solutions, Vol. 4, No. 3, pp. 1-10, 2001.

[5] M. Tang, Y. Feng, L. Burnett, R. Hayward, and K. Raymond, "A reference station placement scheme in deployment of network real-time kinematic positioning systems," Proceedings International Global Navigation Satellite Systems Society Symposium, Sydney, pp. 123-132, December 2007.

[6] M. Tang and Y. Feng, "Area-oriented reference station placement for network RTK," Proceedings International Conference on Computer Science and Software Engineering, Wuhan, December 2008.

[7] M. Garey and D. Johnson, "Computers and intractability: A guide to the theory of NP-Completeness," W. H. Freeman, San Francisco, 1979. 